School of Medicine, Atlanta, Georgia, ${ }^{8}$ Healthcare-Associated Infections Program, California Department of Public Health, Richmond, California

Session: 131. Bacteremia and Endocarditis

Friday, October 5, 2018: 12:30 PM

Background. Staphylococcus aureus treatment guidelines are being revised to include proposed quality measures for evaluation of patients with $S$. aureus bacteremia (SAB) (e.g., infectious disease [ID] consultation, echocardiogram, and documenting clearance of bacteremia). We describe current management practices of SAB to identify opportunities for quality improvement.

Methods. We conducted a pilot assessment of SAB cases reported to CDC's Emerging Infections Program active, laboratory- and population-based surveillance from 24 hospitals in four states during 1-2 months in 2017 or 2018. An SAB case was the isolation of $S$. aureus from a blood culture among adults ( $\geq 18$ years) in the catchment area. We collected clinical and demographic information and performed a descriptive analysis of management of SAB cases.

Results. Among 109 SAB cases identified, 50 (46\%) were methicillin-resistan S. aureus (MRSA). While hospitalized, $87(80 \%)$ patients were evaluated by ID consultation, 90 (83\%) underwent an echocardiogram (26 were transesophageal), and $92(84 \%)$ had documented clearance of bacteremia. During the hospitalization, 15 (14\%) died and 12 (11\%) left against medical advice (AMA). Of those who survived and did not leave AMA, median duration of hospitalization after initial culture was 10.5 days (interquartile range $7-18$ ). In total, 10 survivors ( $9 \%$ of cases) completed at least 2 weeks of antibiotics while hospitalized, and 65 (60\% of cases) were discharged on antibiotic therapy. Among the 25 MRSA patients discharged on antibiotics, common treatments were vancomycin $(64 \%)$, daptomycin $(8 \%)$, ceftaroline $(8 \%)$, and linezolid (4\%). Among the 40 methicillin-susceptible SAB patients discharged on antibiotics, cefazolin (56\%), ceftriaxone (13\%), cefepime (5\%), linezolid (5\%), nafcillin $(3 \%)$, and vancomycin (3\%) were most common. The remainder of outpatient treatments included oral $\beta$-lactams, clindamycin, doxycycline, levofloxacin, and erythromycin.

Conclusion. Overall, the majority of patients with SAB underwent evaluation according to the proposed quality measures and received therapy with targeted anti-staphylococcal agents, although opportunities to optimize treatment remain Hospitalized patients who leave AMA represent a particular challenge for effective SAB therapy.

Disclosures. All authors: No reported disclosures.

\section{Risk Factors for Recurrent Staphylococcus aureus Bacteremia}

Seong-Ho Choi, MD, $\mathrm{PhD}^{1,2}$; Felicia Ruffin, MSN, RN ${ }^{3}$; Lawrence Park, $\mathrm{PhD}^{3}$; Batu K Sharma-Kuinkel, $\mathrm{PhD}^{1}$; Maria Souli, MD PhD ${ }^{4}$; Bobby Warren, MPS Brenda Hansen, $\mathrm{MS}^{5}$; Felix Mba Medie, $\mathrm{PhD}^{6}$ and Vance G. Fowler Jr., $\mathrm{MD}^{7} ;{ }^{1}$ Duke University Medical Center, Durham, North Carolina, ${ }^{2}$ Chung-Ang University Hospital, Chung-Ang University College of Medicine, Seoul, Korea, Republic of (South), ${ }^{3}$ Division of Infectious Diseases, Duke University Medical Center, Durham, North Carolina, ${ }^{4}$ th Department Internal Medicine, National and Kapodistrian University of Athens, School of Medicine, Athens, Greece, ${ }^{5}$ Medicine, Duke University Medical Center, Durham, North Carolina, ${ }^{6}$ Duke University Medical Center, Durham, North Carolina, ${ }^{7}$ Duke University, Durham, North Carolina

\section{Session: 131. Bacteremia and Endocarditis}

Friday, October 5, 2018: 12:30 PM

Background. Recurrent Staphylococcus aureus bacteremia (Re-SAB) occurs in $2-17 \%$ of patients with $\mathrm{SAB}$ within 3-12 months after resolution of the first episode. The risk factors for Re-SAB are incompletely understood.

Methods. Re-SAB was defined as a second episode of SAB after the resolution of the first episode occurring at least 14 days from the date of the last positive blood culture of the first episode. Using the SAB Group Prospective Cohort Study (SABGPCS) data between January 2008 and May 2015, patients with Re-SAB were selected and compared with those without it. Pulsed-field gel electrophoresis (PFGE) was done for the clinical isolates from the Re-SAB group, and spa typing was for those from both groups. Baseline sera from patients with Re-SAB and age/race/gender matched (1:1) control subjects with SAB but without Recurrence underwent Luminex multiplex cytokine array.

Results. Seventy patients experienced Re-SAB (9.3\%) and $686 \mathrm{SAB}$ patients did not. In the Re-SAB group, 156 episodes of SAB were observed. Median time to Re-SAB was 147.5d (IQR, 76-358). Among 65 PFGE-analyzed pairs of isolates from the first and the subsequent episodes, the time to Re-SAB of $<300$ days was more commonly found in the PFGE-identical pairs than in the PFGE-different pairs (75.6\% vs. $33.8 \%$, $P=0.001)$. In the comparison of clinical factors between $56 \mathrm{Re}-\mathrm{SAB}$ patients with available data and 686 without Re-SAB, African American race, dialysis dependence, the presence of foreign body, persistent bacteremia, metastatic abscess formation, and methicillin-resistant $S$. aureus (MRSA) were more frequently observed in patients with Re-SAB. In a multivariate analysis to identify risk factors for Re-SAB, African American race, dialysis dependence, metastatic abscess formation, and MRSA were independent risk factors. The distribution of spa types between the two group was presented in Figure 1.

Conclusion. Re-SAB involves a combination of multiple factors of host, microbe, and treatment. Further laboratory investigation for any determinants in host and microbe is required.
(\%) with Re-SAB $\square$ without Re-SAB

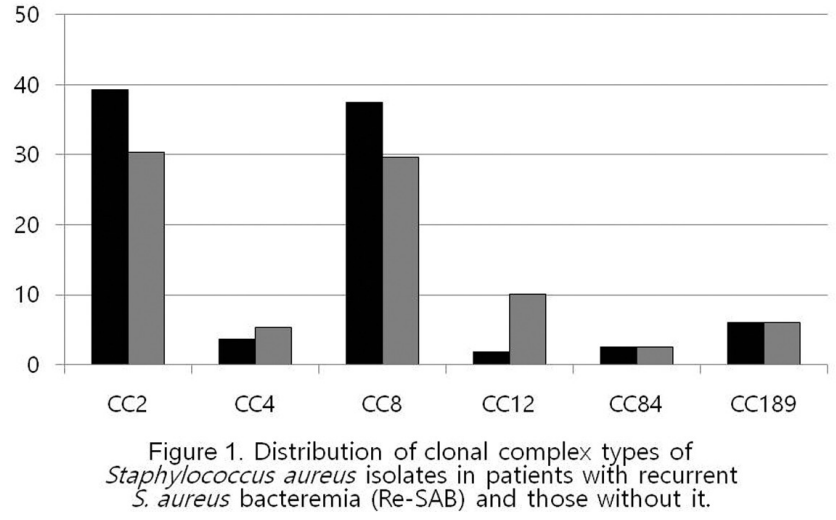

Disclosures. V. G. Fowler Jr., Merck, Cerexa/Actavis, Pfizer, Advanced Liquid Logis, NIH, MedImmune, Basilea, Karius, Contrafect, Regneron, Genentech, Affinergy, Locus, Medical Surface, Inc., Achaogen, Astellas, Arsanis, Bayer, Cubist, Debiopharm, Durata, Grifols, Medicines Co, Novartis: Collaborator, Consultant and Scientific Advisor, Consulting fee, Research grant and Research support.

1061. Comparison of the Acute Physiology and Chronic Health Evaluation (APACHE) II Score and the Pitt Bacteremia Score to Predict Mortality in Methicillin-Resistant Staphylococcus aureus Bacteremia Sarah Jorgensen, PharmD, BCPS, AAHIVP ${ }^{1}$; Evan J. Zasowski, PharmD, MPH ${ }^{1,2}$, Trang D. Trinh, PharmD, MPH ${ }^{1,3}$; Abdalhamid M. Lagnf, $\mathrm{MPH}^{1}$; Sahil Bhatia, BS ${ }^{1}$ and Michael J. Rybak, PharmD, MPH, $\mathrm{PhD}^{4} ;{ }^{1}$ Anti-Infective Research Laboratory, Department of Pharmacy Practice, Wayne State University, Eugene Applebaum College of Pharmacy and Health Sciences, Detroit, Michigan, ${ }^{2}$ Department of Pharmacy Practice and Translational Research, University of Houston College of Pharmacy, Houston, Texas, ${ }^{3}$ Department of Clinical Pharmacy, University of California, San Francisco, School of Pharmacy, San Francisco, California, ${ }^{4} 259$ Mack Ave, Suit 4131, Eugene Applebaum College of Pharmacy and Health Sciences Bldg, 259 Mack Ave, Detroit, Michigan

Session: 131. Bacteremia and Endocarditis

Friday, October 5, 2018: 12:30 PM

Background. Methicillin-resistant Staphylococcus aureus bloodstream infection (MRSA BSI) is associated with high morbidity and mortality. The prediction of outcomes may have a profound impact on clinical decision making and risk stratification. The Acute Physiology and Chronic Health Evaluation (APACHE) II Score and the Pitt Bacteremia Score (PBS) have been repeatedly described as independent predictors of mortality in MRSA BSI. The APACHE II is complex to calculate and many of the variables may not be pertinent to MRSA BSI. The PBS is a simple score using readily assessable variables. The comparative predictive performance of the two models in MRSA BSI has not been evaluated.

Methods. Retrospective, observational, singe-center cohort study in adults with MRSA BSI between 2008 and 2018. Patients who did not receive active therapy $\leq 72$ hours of index culture were excluded. APACHE II and PBS were calculated using the worst physiological values recorded $\leq 24$ hours of blood culture collection. Discriminatory ability for 30-day mortality was assessed using the c-statistic and was compared using the Hanley and McNeil method. The best cut-off point in each scoring system was determined using the Youden Index $(\mathrm{J})$.

Results. A total of 455 patients were included. The median (IQR) PBS and APACHE II were $2(0,3)$ and $18(11,23)$, respectively. All-cause 30 -day mortality was $16.3 \%$. The c-statistic ( $95 \% \mathrm{CI})$ for the APACHE II vs. PBS in the overall cohort and stratified by ICU status were: $0.813(0.763,0.863)$ vs. $0.717(0.653,0.782), P=0.0035$ ICU $0.729(0.610,0.848)$ vs. $0.570(0.442,0.699), P=0.026$; and non-ICU $0.821(0.761$, $0.881)$ vs. $0.700(0.614,0.786), P=0.0046$, respectively. The APACHE II with the maximum $J$ value was 21 ; sensitivity, specificity, positive predictive value (PPV), and negative predictive value (NPV) for 30 -day mortality were $81.08 \%, 72.97 \%, 36.81 \%$, and $95.21 \%$, respectively. The PBS with the maximum J value was 3; sensitivity, specificity, PPV, and NPV were $66.22 \%, 72.18 \%, 31.61 \%$, and $91.67 \%$, respectively.

Conclusion. The APACHE II was superior to the PBS in predicting 30-mortality in patients with MRSA BSI in the overall cohort and stratified by ICU status at BSI onset. Future research to develop a more practical scoring model with high discriminatory power is needed.

Disclosures. M. J. Rybak, Allergan: Consultant, Grant Investigator and Speaker's Bureau, Research grant and Research support. Achaogen: Consultant, Grant Investigator and Speaker's Bureau, Consulting fee, Research grant and Research support. Bayer: Consultant, Grant Investigator and Speaker's Bureau, Consulting fee, Research grant and Research support. Melinta: Consultant, Grant Investigator and Speaker's Bureau, Consulting fee, Research grant and Research support. Merck: Consultant, Grant Investigator and Speaker's Bureau, Consulting fee, Research grant 